\title{
Etude de la qualité physico-chimique et bactériologique dans les plans d'eau portuaires
}

Mounia El Haji

Etudiante en thèse, Laboratoire de Mécanique, GRGC, Université du Havre.

Claude LOUTS

Professeur des Universités, Laboratoire de Mécanique, GRGC, Université du Havre.

Résumé:

L'étude à réaliser sur ce sujet s'inscrit dans le domaine des recherches pratiques visant à déceler un certains nombres de relations entre les micro-organismes et les divers facteurs ambiants, physiques, chimiques et biologiques dans les milieux en voie de dystrophie et spécialement dans les plans d'eau divers, à niveau constant ou variable et à salinité allant de l'eau saumâtre à l'eau de salinité normale.

L'importance de certaines bactéries d'anaérobioses dans l'attaque par corrosion incite à faire un couplage de processus bactériens et physico-chimiques en des sites soumis à des phénomènes d'eutrophisation dans des conditions de développement et l'action de ces micro-organismes.

Les mico-organismes jouent un rôle considérable dans la nature en intervenant dans de multiples cycles biologiques. D'importantes dégradations ayant été observées au niveau des ouvrages en acier. Pour ce faire on examine l'intervention des bactéries du cycle du soufre.

Au cours de la minéralisation de la biomasse morte, le soufre, comme les éléments essentiels (carbone, oxygène, azote), se trouve sous différentes formes, soit lié à différents composés organiques, soit libre, sous forme inorganique. En conditions anaérobies, le sulfure est la forme finale et énertiquement stable du cycle du soufre.

Le but de cette étude est de rechercher les variations physico-chimiques des milieux relativement clos dans lesquels ils se développent ainsi que les fluctuations des populations bactériennes productrices d'acide sulfurique qui en résulte. De cette façon, on pourra également apporter une contribution par une approche bactérienne à la compréhension des phénomènes de durabilité des ouvrages immergés dans les zones portuaires. 


\section{Introduction}

L'objectif de l'étude est de comprendre le fonctionnement des systèmes d'eau stagnants dans la zone industrielle du Havre, afin de déterminer les principaux facteurs responsables de l'élimination de l'azote et du phosphore ainsi que la corrosion bactérienne et son impact sur les installations fixes portuaires. Une analyse des variables climatiques, de la matière organique et des formes minérales de l'azote et du phosphore a montré l'évolution saisonnière marquée d'où l'adaptation de l'écosystème à la dégradation de la matière organique.

L'élimination de l'azote et du phosphore est liée principalement à l'activité algale, qui entraîne une assimilation biologique et une élévation de $\mathrm{pH}$, responsable des phénomènes chimiques de volatilisation de l'azote ammoniacal et de précipitation de phosphate de calcium.

La biomasse algale dans ces systèmes est étroitement liée aux conditions climatiques, à la charge organique et au temps de rétention dans les bassins.

Le but donc de l'étude est lã compréhension du comportement des nutriments dans ces bassins ainsi que la mise en évidence de bactéries responsables de la corrosion des structures fixes dans le port du Havre.

\section{Présentation et analyses générales}

\section{1. Présentation de la zone d'étude}

Le port du Havre est situé à la pointe du Pays de Caux, au fond de la baie de Seine en Manche orientale. Il s'étend parallèlement à l'estuaire de la Seine dont il est isolé, sur un substratum d'alluvions quaternaires de ce fleuve qu'il rejoint par un canal, le canal de Tancarville long d'environ $25 \mathrm{~km}$. La jonction avec la Seine se fait à l'est à la hauteur de Tancarville (SeineMaritime) par une écluse. La partie occidentale du canal de Tancarville reçoit les eaux d'une rivière, la Lézarde. Entre le canal de Tancarville et la Seine s'étend une zone industrielle, avec principalement des industries pétrochimiques, automobiles, métallurgiques et une cimenterie (Breton, 1981). Ces industries sont desservie par le grand canal du Havre plus large que le canal de Tancarville (figure 1).

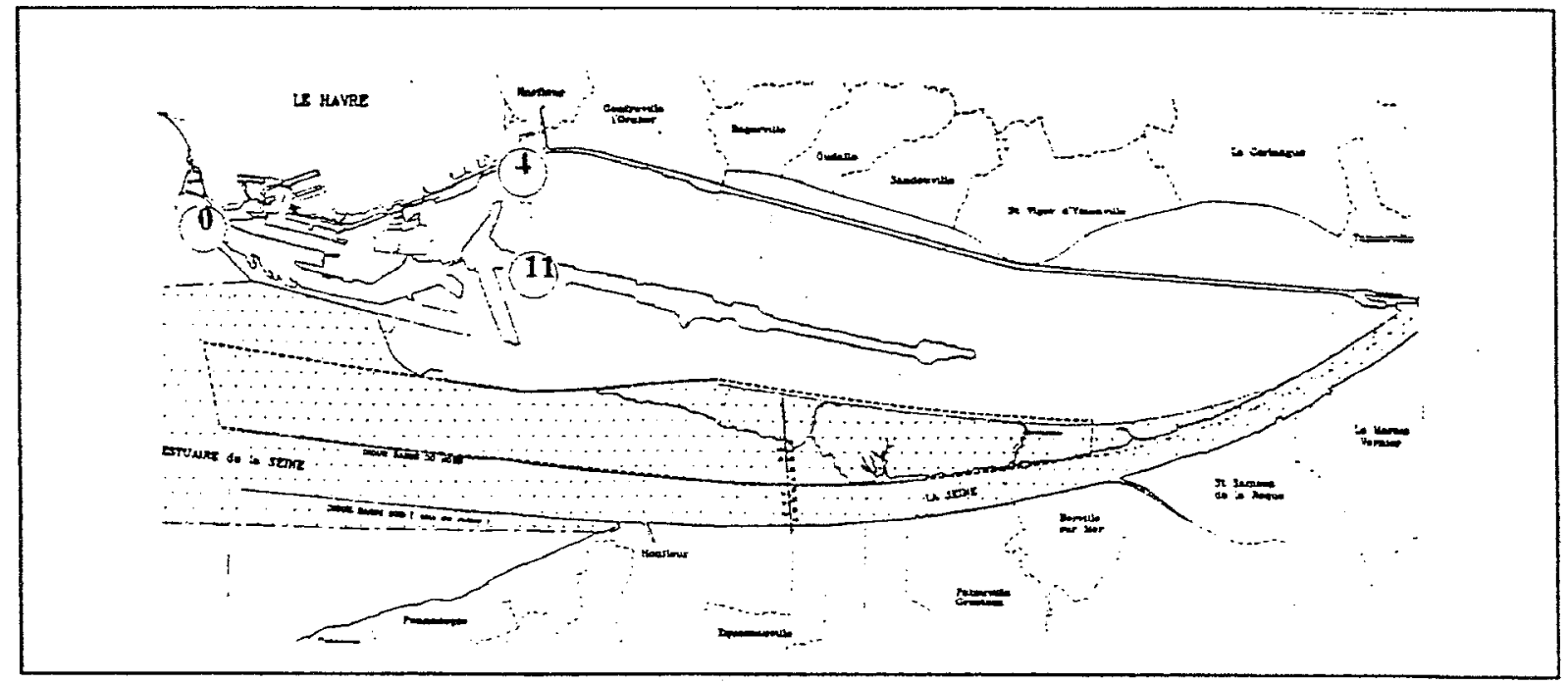

Figure 1: Présentation de la zone d'étude 
La zone d'extension du port du Havre fait l'objet de contrôles sur la qualité des eaux de bassins. Depuis 1977, onze points de prélèvement sont suivis mensuellement en ce qui concerne les paramètres de qualité générale : température, turbidité, $\mathrm{pH}, \mathrm{MES}$, salinité, oxygène dissous, ammonium, azote kjeldahl, nitrites, nitrates, etc...

L'ensemble des mesures réalisées ces 20 dernières années constitue une base de données permettant de caractériser et d'expliquer les tendances évolutives de la qualité des eaux des bassins, ce qui permettra d'analyser et de comprendre les phénomènes de corrosion des ouvrages immergés du port.

\section{2. Analyse de la base de données : tendances évolutives (figure 2)}

En fonction de la variation des paramètres analysés une interprétation en fonction des saisons a été réalisée comme suit :

\subsection{De septembre à février}

Les mois de septembre et d'octobre sont marqués par une mortalité rapide et importante du phytoplancton (CREOCEAN, 1993). Les concentrations maximales sont obtenues dès le mois septembre-octobre pour les phosphates provenant de l'hydrolyse de la matière organique, alors que les stocks d'azote sont reconstitués plus tardivement : en novembre et décembre pour l'ammonium et en février pour les nitrates.

Pendant toute la période de dégradation de la matière organique, l'activité bactérienne est fortement consommatrice d'oxygène, d'où l'enregistrement des plus basses concentrations en oxygène dissous de l'année.

\subsection{2. de mars à août}

Le développement du phytoplancton semble avoir lieu dès le mois de février. C'est le début d'une baisse des concentrations en sels nutritifs : Silicates, ammonium et nitrates. Ce développement permet une bonne oxygénation de l'eau des bassins au printemps (avril et mai) et en période estival. Passée la première poussée printanière, un renouvellement régulier des populations de phytoplanctons a lieu. L'âfflux de la matière organique végétale morte passe en solution sous forme ainmoniacale et est immédiatement consommé par la «deuxième génẻration» du phytoplancton.

Le mois d'août est marqué par les plus fortes températures annuelles. Les concenîirations en fluorures et la salinité atteignent leur maximum. Dès le mois de septembre débute un nouveau cycle avec la disparition massive du phytoplancton.

\section{Approche bactériologique de la corrosion des ouvrages immergés}

\subsection{Généralités}

Il est reconnu à l'heure actuelle que des dégradations très importante des structures, même avec les matériaux de haute résistance, sont dues en partie à l'action de micro-organismes (Baril, 1995). L'eau contient toujours des bactéries susceptibles de se développer directement dans le milieu ambiant, soit capables d'y végéter et d'attendre que des conditions favorables soient réalisées pour proliférer. C'est pourquoi certaines installations, fonctionnant parfaitement lors de leur mise en service, se trouvent, après un temps plus ou moins long, perturbées par des accidents tels que pertes de charge, perforations, obstructions, etc... 


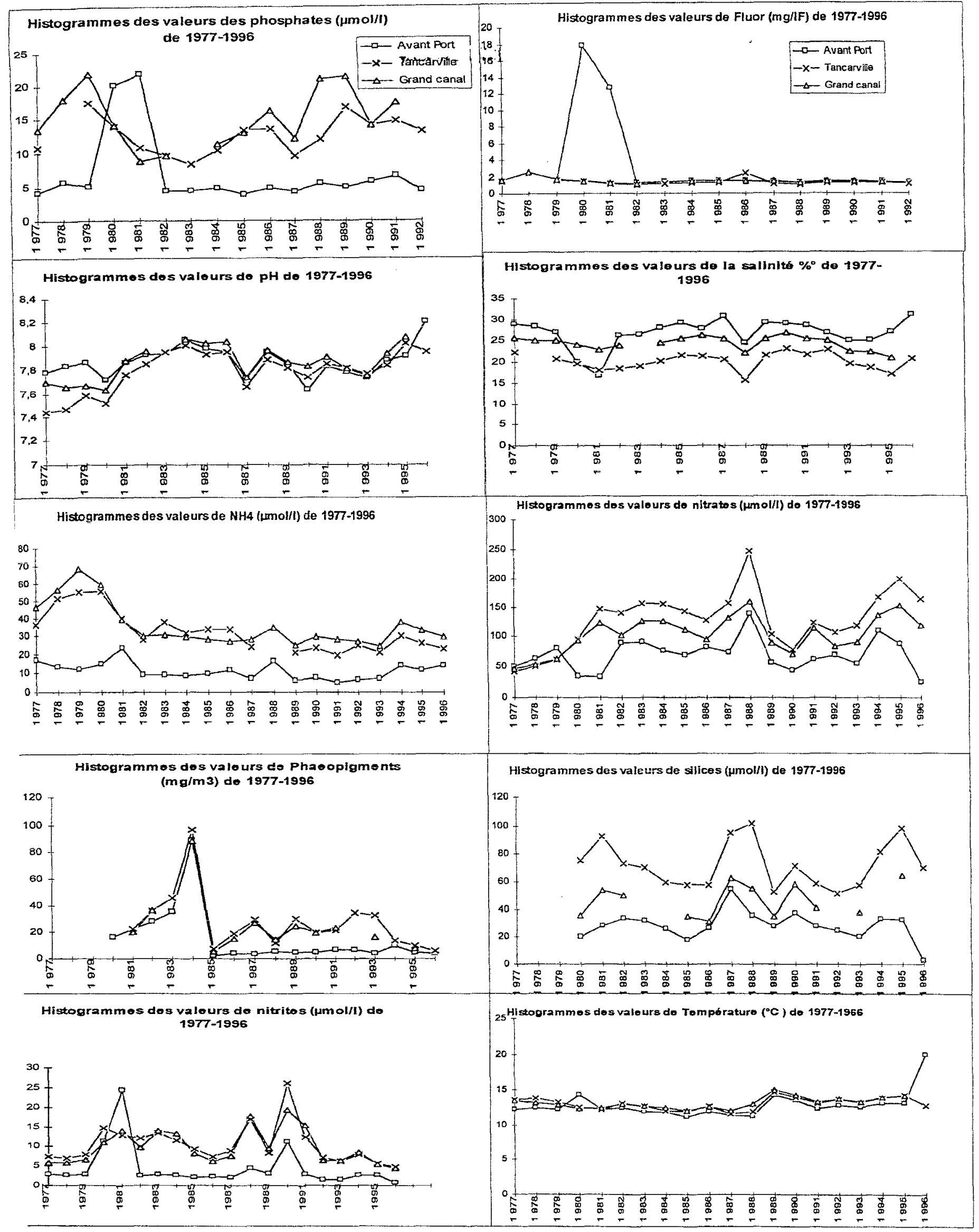

Figure 2 : Tendances évolutives des paramètres physico-chimiques 
Il est à noter que pour une eau dont la composition chimique est relativement constante et ne varie que dans certaines limites, sa pollution bactériologique est par contre excessivement variables, soit par le nombre, soit par la qualité des bactéries.

Ce sont essentiellement les bactéries sulfato-réductrices (avec la contribution des ferrobactéries et des sulfobactéries) qui, en produisant des sulfures et consommant les sulfates, s'attaquent aux structures fixes immergés (Chantereau, 1977).

\section{2. Contexte théorique}

L'hydrogène gazeux formé sur les surfaces cathodiques s'élimine en se combinant avec l'oxygène libre ou dissous dans le milieu ambiant. En l'absence d'oxygène, l'hydrogène persistant sur la surface métallique polarise le système et arrête l'effet de pile. Les réactions peuvent s'écrire de la manière suivante:

$$
\begin{aligned}
& \mathrm{H}_{2} \mathrm{O} \Longrightarrow \mathrm{H}^{+}+\mathrm{OH}^{-} \\
& \mathbf{2} \mathbf{H}^{+}+\mathbf{2} \mathbf{e}^{-} \rightleftharpoons \mathbf{H}_{2}
\end{aligned}
$$

Si l'eau contient des bactéries sulfato-réductrices, elles consomment l'hydrogène et il y a naissance d'un courant galvanique. Ces bactéries sont capables de réduire les sulfates en sulfures selon la réaction globale :

$$
4 \mathrm{Fe}+\mathrm{SO}_{4}{ }^{2-}+2 \mathrm{H}^{+}+2 \mathrm{H}_{2} \mathrm{O}=3 \mathrm{Fe}(\mathrm{OH})_{2}+\mathrm{FeS}
$$

facilitant l'oxydation du fer et provoquant sa saturation particulièrement néfaste.

\section{Démarche expérimentale}

Des prélèvements ont été réalisé en trois sites de la zone industrielle du port du Havre. Ces points d'échantillonnages sont recensés parmi ceux des analyses en continu depuis 1977. Ces points se veulent représentatifs des 3 types de bassins existant sur la zone portuaire:

- Bassin à niveau constant dans le canal de Tancarville (point 4),

- Bassin influencés par la marée dans la zone de l'avant port(point 0),

- Bassins à flot et jusant : marnage intermédiaire dans le grand canal du Havre (point 11).

Des mesures de $\mathrm{pH}$, de salinité et de matière organique (dans le sédiment) on tété récalisées en chaque point en trois hauteurs différentes : en surface, mi-profondeur et au fond. Far ailleurs, les échantillons prélevés ont permis de déterminer en laboratoire la concentration en azote kjeldahl (NTK)

\section{Protocoles expérimentaux}

\section{1. Salinité}

L'analyse a été faite au réfractomètre. Quelques gouttes d'eau sont déposées dans le réceptacle de l'appareil est la lecture se fait, directement dans l'oculaire par visualisation d'une échelle graduée.

\section{2. Azote kjeldahl}

L'azote kjeldahl correspond à l'azote présent sous forme organique et ammoniacale, à l'exclusion des formes nitreuse et nitrique. En d'autre termes, il s'agit de formes non oxydé de l'azote.

Le dosage de l'azote se décompose en deux phase : digestion et distillation (Rodier, 1984). 
La digestion consiste dans un premier temps à analyser la matière azotée par de l'acide sulfurique à chaud, pour former du sulfate d'ammonium. Cette opération nécessite des réactifs: le sulfate de potassium permet d'élever le point d'ébullition du mélange et de sulfate de cuivre et le sélénium sont sollicités comme catalyseurs.

La distillation permet de déplacer par la soude l'ion ammonium qui est ensuite entraîné à la vapeur d'eau puis fixé dans l'acide borique. L'ammoniaque est alors dosé directement par l'acide sulfurique en présence d'un indicateur coloré.

\section{Interprétation des résultats}

La courbe de concentration en azote kjedahl laisse apparaître une tendance à la diminution de ces concentrations en profondeur (Figure 3 ).

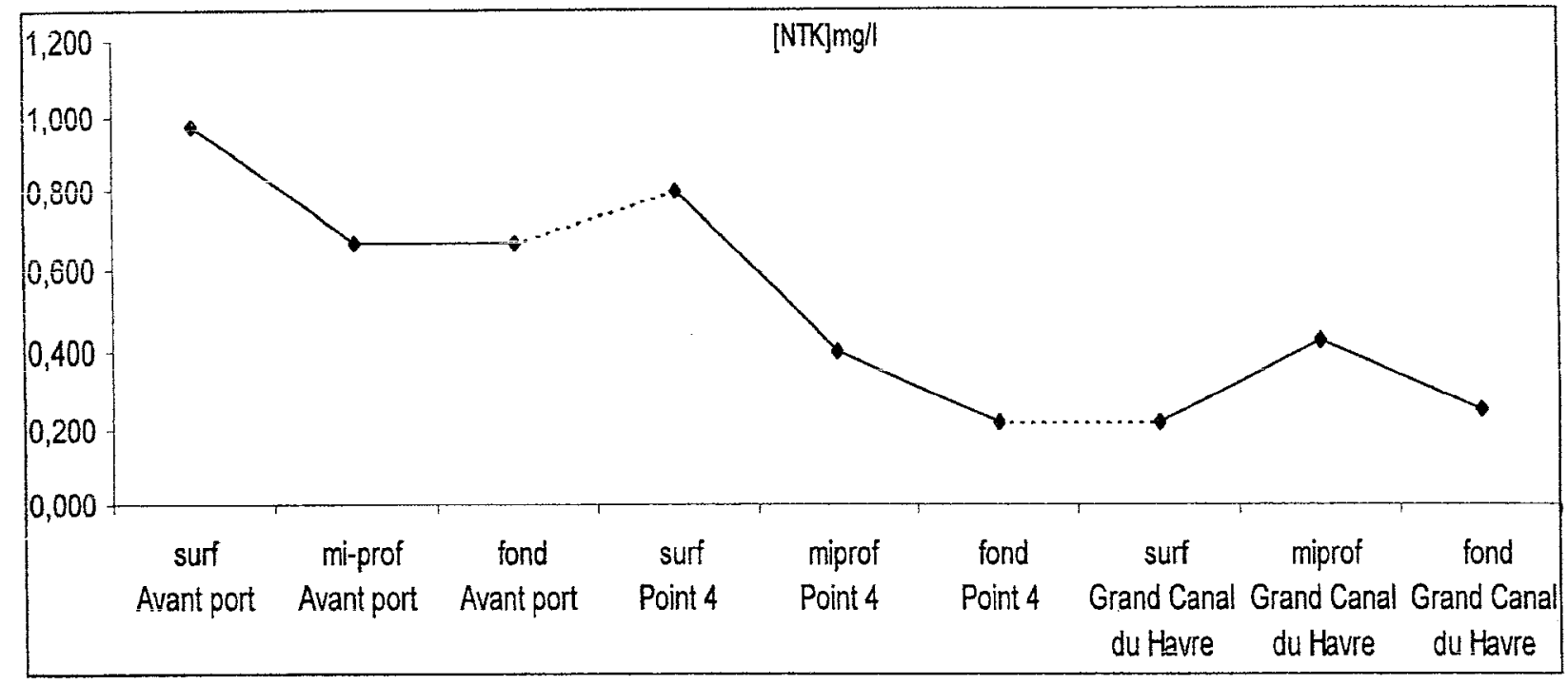

Figure 3: Courbe des concentrations en [NTK] en mg/l

En considérant le fait que les formes azotées sont les accepteurs finaux d'électrons dans le cycie de corrosion par voie bactérienne, leur diminution se traduit par leur utilisation par les microorganismes. Ces formes azotées seront transformées en azote atmosphérique.

En ce qui concerne le taux de salinité, il est représentatif des eaux saumâtres, résultat d'un mélange d'eau douce et d'eau marine. Ces eaux sont isolées dans des systèmes confinés donc la salinité est plus faible qu'au large. Par ailleurs subissent l'influence de l'eau douce apportée par des affluents comme la Lézarde qui se jette dans le canal de Tancarville.

Une salinité élevée conditionne un milieu acide. Dans les bassins du port du Havre, les taux de salinité relativement faibles autorisent un $\mathrm{pH}$ compris entre 6.5 et 8 . Il s'agit d'un $\mathrm{pH}$ idéal pour le développement bactérien (figure $4 \mathrm{a}$ et $4 \mathrm{~b}$ ).

Par ailleurs les sédiments déposés au fond des bassins du port du Havre. sont à dominance argileuse et siliteuse (Goutierre, 1987).

Il s'agit de sédiments fins qui favorisent une évolution vers un système de floculation. Ce système constitue un support fixe pour la matière organique. Celle-ci constitue un élément précurseur à l'origine des cycles de phosphore et d'azote indispensables au développement de tout système 


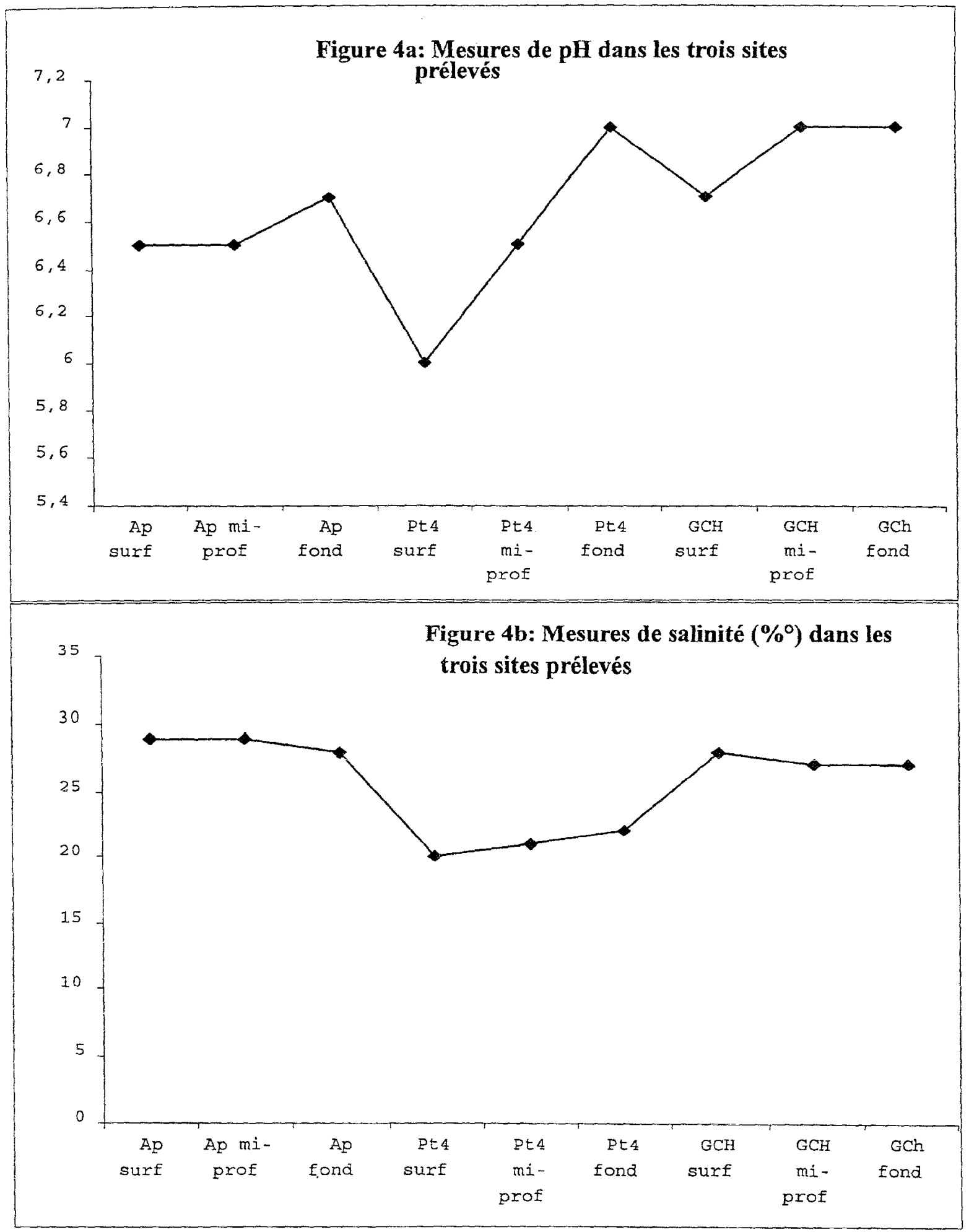


faunistique et floristique. Les analyses réalisés dans les bassins du port du Havre laissent apparaître des teneurs relativement élevées de la matière organique. Ainsi plus le taux de matière organique est élevé, plus la probabilité d'apparition de bactéries d'anaérobiose est élevée.

Autres paramètres significatifs: la turbidité et la matière en suspension. Ces deux paramètres augmentent de la surface vers le fond et diminuent lorsqu'on passe de l'hiver à l'été avec un pic au printemps correspondant au bloom phytoplanctonique.

Ce phénomène favorise une forte production en biomasse algale qui engendre une production en oxygène dissous, une baisse de la concentration en $\mathrm{CO}_{2}$ et la mise en place des cycles d'azote, de sulfate et de phosphate. Dans ce cas, une augmentation de la turbidité au fond de bassin autorise le processus de floculation, support de la matière organique pour les bactéries.

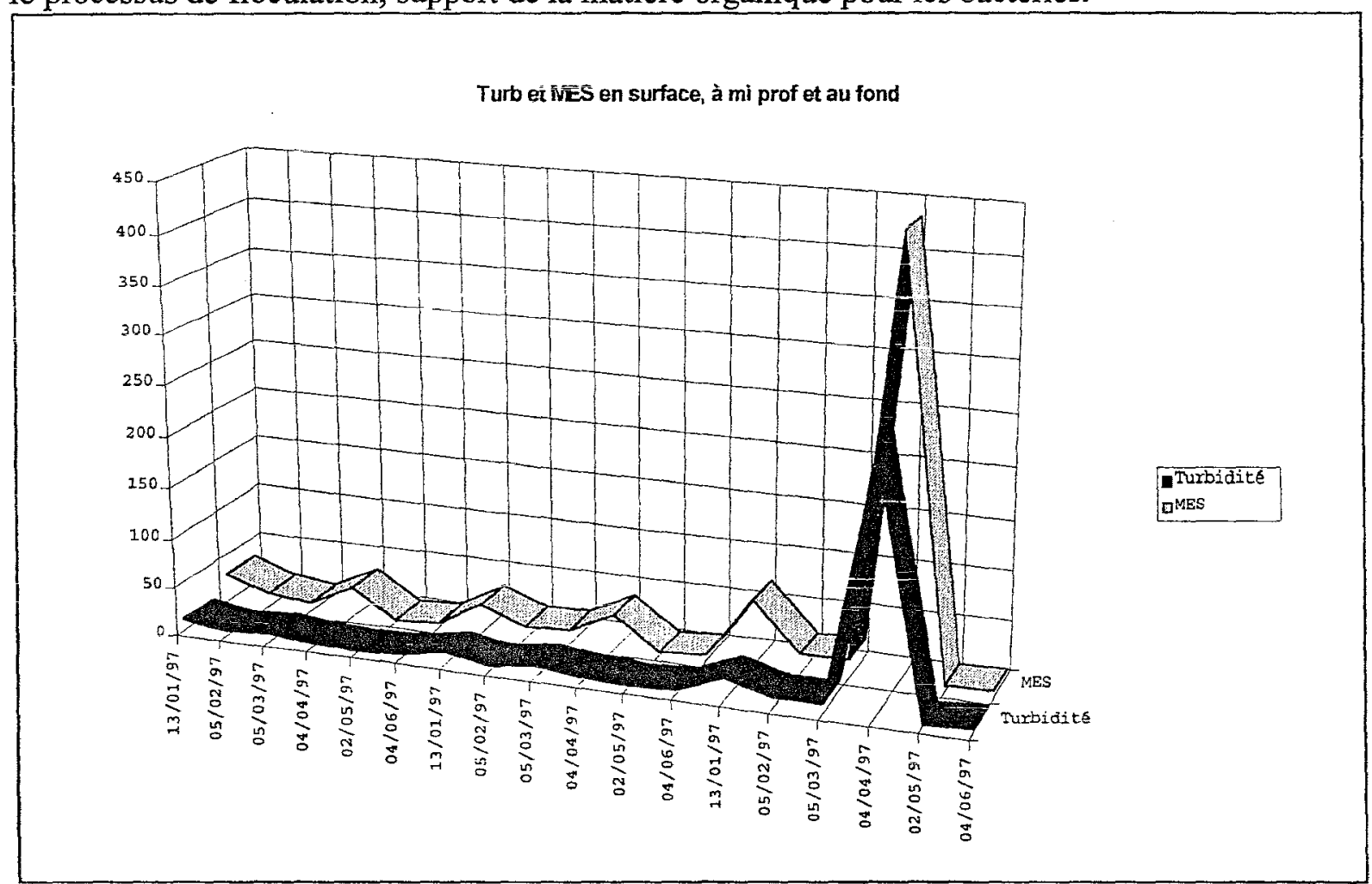

Figure 5: Evolution de la turbidité (NTU) et des MES (mg/l) en surface, mi-profondeur et au fond de la zone de l'avant port du Havre.

\section{Conclusion}

Les taux de $\mathrm{pH}$ et de salinité à différentes hauteurs en un même point mettent en évidence une stratification du système aquifère. Ceci permet de faire une remarque sur la méthodologie utilisée pour les prélèvements ayant permis l'élaboration de la base de données sur les 20 dernières année: ces bassins ont des profondeurs différentes et des prélèvements à 1,5 mètre sous la surface de l'eau ne permettent pas une comparaison rigoureuse entre les points.

Ainsi, au regard des moyens mis en oeuvre par le port du Havre pour le suivi de la qualité des eaux et de bassins, il semblerait intéressant de réaliser des contrôles à différentes hauteurs, ou 
pour le moins de les réaliser sur une base de référence commune, par exemple un référentiel d'altitude situé au fond du bassin.

Dans les zones portuaires où l'eau de mer est plus ou moins polluée par les effets des produits industriels ou d'effluents urbains, en présence de micro-organismes a une influence plus ou moins non négligeable sur les phénomènes de corrosion bactérienne. L'apport en matières organiques permet l'augmentation des bactéries d'anaérobiose susceptibles de modifier les conditions d'oxygénation des sédiments et favoriser le phénomène de corrosion par ces bactéries.

\section{Références}

[1] Baril J. C. (1995). Digues portuaires et protection du littoral : Entretien au service des études et travaux d'infrastructure du Port Autonome du Havre. 46 p.

[2] Benbouzid-Rollet N. (1993). Influence des bactéries sulfato-réductrices sur la corrosion d'acier en milieu marin : étude au laboratoire et en milieu naturel. Thèse, Université de Brest. $180 \mathrm{p}$.

[3] Breton G. (1981). Observations sur l'écologie et les peuplements des bassins du port du Havre (France). Bull. Trim. Soc. Géol. Norm.et Amis du Muséum du Havre. p, 46. Fig. 2, p. 55.

[4] Buslov V.M., Rojansky M. (1989). Assessment of corrosion damage to concrete marine structures. FIP Notes, (2), pp. 8-12.

[5] Chantereau J. (1977). Corrosion Bactérienne. Technique et Documentation. 246 p.

[6] Collee R. (1975). Corrosion Marine. Editions CEBEDOC. 192 p.

[7] Etude de la qualité des plans d'eau du Havre : Analyse des résultats obtenus entre 1977-1992. CREOCEAN. Octobre (1993), dossier 32106.55 p.

[8] Goutierre I. (1987). Nouvel appareil de cisaillement circulaires pour argiles très peu dense in situ: Utilisation pour l'étude de la navigabilité dans les chenaux envasés. Thèse de doctorat. $175 \mathrm{p}$.

[9] Flux de pollution dû à la zone industrielie et portuaire du Havre : Port Autonome du Havre. Octobre (1975). $17 \mathrm{p}$.

[10] La protection contre la corrosion des structures métalliques immergées : Service Technique Central Des Ports Maritimes Et Voies Navigables. (1988). Notice STC. QG n ${ }^{\circ}$ 88.2. 258 p.

[11] Rodier J. (1984). L'analyse de l'eau, eaux naturelles, eaux résiduaire et eaux de mer: physico-chimie, bactériologie, biologie. 8 édition Dunod, Paris. 